\title{
IDENTIDAD, ESTILO Y SUBCULTURA EN PEREIRA: LA MODA/VESTIDO EN EL METAL, PUNK Y HIP HOP
}

\author{
IDENTITY, STYLE AND SUBCULTURE IN PEREIRA: \\ FASHION/DRESS IN THE METAL, PUNK AND HIP HOP
}

\section{NATALIA URIBE RESTREPO}

Diseñadora de Modas. Estudiante de maestría en Estudios Humanísticos. Docente de planta en la Fundación Universitaria del Área Andina Seccional Pereira. nuriber@funandi.edu.co. Grupo de investigación ESFERAS, línea de investigación Historia, contexto y cultura

\begin{abstract}
RESUMEN:
La moda/vestido desempeñan numerosas funciones en la vida del ser humano; una de las más significativas es el uso de la misma como vehículo de expresión de identidad, la cual permite reconocer cómo un usuario se identifica de manera tanto individual como colectiva, además de mostrar las diferentes dinámicas del contexto donde este se mueve. Cumplen una de las funciones más representativas es en la relación moda/ vestido e identidad que se da en las subculturas. Sin embargo, y a pesar de su relevancia, no se encuentran suficientes estudios relacionados con esta relación dentro de contextos colombianos específicos, y mucho menos desde la perspectiva del diseño. Así pues, para enfrentar esta problemática se llevó a cabo una investigación de corte cualitativo-etnográfico en la ciudad de Pereira con los tres grupos subculturales más representativos en la ciudad para comprender los significados del uso de sus elementos de vestir.
\end{abstract}

\section{PALABRAS CLAVE:}

Moda/vestido, identidad, subculturas, metal, hip hop, punk.

\section{ABSTRACT:}

Fashion/dress plays numerous functions in the life of the human being; one of the most significant is the use of it as vehicle of expression of identity, because it allows to recognize how the user identifies of both in an individual and collective way, beside showing the different dynamics of the context where it moves. Being like that, where it accomplishes one of the most representative functions is in the relation fashion/dress and identity that is given in the subcultures. Nevertheless, and in spite of its relevancy, there are not sufficient studies related to this relation inside Colombian specific contexts, and much less from the perspective of the design. That is why, to face this problematic, there was conducted a qualitative - ethnographic research in the city within the three most representative subcultural groups in the city, with the aim of understand the meanings of the use of dress elements.

\section{KEYWORDS:}

Fashion/dress, identity, subculture, metal, hip hop, punk. 


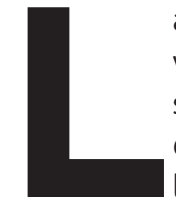

a moda/vestido ${ }^{1}$ es una parte fundamental de la vida del ser humano, ya que desde siempre desempeña numerosas funciones que van desde la comunicación no verbal, la decoración, el pudor, la protección y la expresión de identidad.

A pesar de la importancia de las funciones mencionadas, hay algunas que son más significativas que otras; tal es el caso de la moda/vestido como vehículo de expresión de identidad, ya que esta relación no solo es una evidencia de la realidad de un contexto, sino que muestra cómo el sujeto puede identificarse individual y colectivamente al mismo tiempo; además de servirle al usuario de herramienta que le otorga pertenencia dentro de la sociedad.

De esta manera, se encuentran varios tipos de prendas que a través del tiempo cumplen importantes funciones como expresión de identidad; por ejemplo: el sastre, la minifalda, las botas militares, entre otros. Si algo tienen en común dichos elementos, es que sus obras más importantes se dan dentro de las Llamadas subculturas urbanas, definidas por Hebdidge (1979) como "grupos que se forman mediante acuerdos comunales y simbólicos con un sistema más grande de la tardía cultura industrial, dentro de contextos históricos y culturales específicos.

Dada la relevancia de las expresiones de identidad mediante la moda/vestido dentro de las subculturas, este tema es objeto de diferentes estudios desde los años 70 por autores como Stuart Hall (1975), Dick Hebdige (1979), Mike Brake (1985), David Muggleton (2000), Hisroshi Narumi (2010), entre otros; los cuales tienen una característica en común: sus resultados son aplicables solo al contexto donde se utilizan las prendas estudiadas.

En el caso de Colombia hay pocas publicaciones acerca del vestuario en la cultura juvenil (más no en la subcultura), como los estudios de perfiles de consumidores de la Cámara de Comercio de Bogotá (2009), en los que se mencionan algunas desde la perspectiva del usuario de moda, consumidor; desde el aspecto comercial o como brote estilístico típico de las etapas de rebeldía juvenil. La interpretación de las mismas se queda corta, ya que las desconocen como reflejo de la realidad del país.

Lo que sucede en Colombia acontece en Pereira, donde la información que existe acerca de estas manifestaciones en la ciudad es casi nula, porque se enmarca en la tendencia nacional y menciona al vestuario como parte de las expresiones de las subculturas; con una explicación superficial y básicamente las mismas que existen en los estudios internacionales; así pues, esta información suele ser desactualizada y descontextualizada, como una que apareció en un periódico local.

Retomando lo anterior, un aspecto que debe tenerse en cuen-

Moda/vestido dentro de los estudios de moda es acuñado por Kawamura (2011) como un todo, ya que son dos perspectivas diferentes pero complementarias, pues la moda es entendida como una entidad cultural y el vestido como las prendas, modificaciones, accesorios, etc ta es la popularidad cada vez mayor de las subculturas en la ciudad y las personas que las integran, como parte de la realidad pereirana que está en permanente cambio y se refleja en ellas.

Aun así no hay un entendimiento de sus prácticas y de los significados de los elementos de moda/vestido que utilizan; por lo cual, gracias a que son representaciones del contexto y ayudan a deducir la realidad de una sociedad y el pensamiento de la misma, se hace necesario estudiarlos y comprender las razones de sus manifestaciones visuales, lo cual además evidencia la relevancia de la moda/vestido dentro de la vida de los individuos y grupos sociales.

Con objeto de conocer y comprender los significados de los elementos de moda/vestido que representan la identidad de los individuos de las subculturas de la ciudad de Pereira, así como la caracterización de los diferentes grupos enfocándose en 3 de ellos: metaleros, punkeros, hip hopers, ubicados en el sector del centro de Pereira, se desarrolló la investigación que respalda esta publicación.

De manera que para el desarrollo de la investigación se tomó el enfoque cualitativo - etnográfico, recurriendo principalmente a las técnicas de observación participante, registro fotográfico y entrevistas no estructuradas con informantes clave. Para esto se escogieron tres subculturas: metaleros, hip hopers y punkeros, ya que cumplían con ciertas características que permitían llevar a cabo el estudio con una muestra representativa, lugares de encuentro y vestuario característico.

\section{CONCEPTOS BÁSICOS}

\section{La identidad}

Un tema importante en esta investigación fue la identidad, ampliamente discutido, usado y reusado en diferentes contextos y distintos propósitos, sobre todo en lo concerniente a la juventud (Buckingham, 2002). El debate acerca de la identidad se da por la palabra en sí: por un lado, esta significa algo único en cada persona que lo distingue de los demás, pero al mismo tiempo implica una relación con un colectivo o un grupo social. Esto se debe que cada persona como ser único es un resultado de quien está con ella y de las situaciones sociales en las que se encuentra, mostrando que no se es libremente lo que se quiere ser, si no que se es un producto de un contexto, exponiendo así la ambivalencia del término y su contradicción. Podría decirse que la identidad es aquella construcción que le da sentido y forma la esencia social del individuo; es el reflejo de sus características individuales y a la vez de las particularidades de los grupos a los cuales pertenece.

\section{Las subculturas}

Uno de los temas asociados con la identidad en la moda/ vestido son las subculturas, porque funcionan en los jóvenes como una vía para pertenecer y definirse .Es importante men- 
cionar que acerca de la subcultura se ha discutido bastante; el término causa debate y es difícil de definir, puesto que se dice que es una forma de organización y reconocimiento de un grupo de valores, comportamientos y acciones que corresponden a una resistencia frente a una serie predominante de normas; pero igualmente se sostiene que no hay definición como tal de subcultura, porque estas manifestaciones no son un subnivel de la cultura reinante sino que son parte de ella. Aun así es importante tener en cuenta que en la actualidad no existe algo como un grupo predominante de normas, ya que hay diferentes corrientes tanto de pensamiento como de estilo, y puesto que la dominancia desaparece no hay algo a lo cual resistirse. Es aquí donde la subcultura se vuelve más una vía para mostrar la identidad y subjetividad, que para resistir, puesto que las subculturas no son formas privilegiadas que se salen del sistema. Estos grupos son una hibridación del contexto: el material educativo disponible y la cultura comercial implican, según Soriano (citado en Ávila-Toscano, Gutiérrez \& Pérez, 2011), que "comparten una identidad asociada a símbolos culturales, como el uso de un determinado peinado, una vestimenta particular, un sistema de creencias y el frecuente consumo cultural asociado a la música y a las representaciones que la misma convoca".

Otra característica importante de la subcultura, consiste en que confecciona su estilo a partir de objetos cotidianos, donde hay una preocupación por el consumo de productos relacionados con la moda/vestido, pues estos tienen gran importancia, ya que son una vía de comunicación intencional con un ordenamiento extremo de significados y un lenguaje preciso (Hebdige, 1979).

\section{El estilo}

En cuanto al concepto del estilo, puede decirse que es "la manifestación simbólica de las culturas juveniles, expresada en un conjunto más o menos coherente de elementos materiales e inmateriales, que los jóvenes consideran representativos de su identidad como grupo". (Feixa, 1996).

La mayoría de las subculturas comparten un estilo interior en el que manifiestan su identidad, tanto colectiva como individual; están conectados a un mercado de consumo específico creado por ellos, ya que seleccionan los artículos que consumen y se transforman a través de las prácticas significantes. Dichos estilos están confeccionados a partir de objetos cotidianos con doble significado (Hebdige, 1979); esto se da ya sea invirtiéndolo o modificándolo. Es importante tener en cuenta que se crea un estilo con la organización activa de objetos, actividades y valores, que producen y organizan una identidad de grupo (Feixa, 1996).

Los nuevos significados de estos estilos emergen porque los fragmentos dispersos que los componen, tomados de aquí y de allá, se integran en un universo estilístico diferente.

\section{La representación cultural}

Otro concepto importante es el de la representación cultural, porque a través de la historia de la humanidad se ha expresado la realidad de formas diversas: pictórica, con objetos, la escritura o la moda/vestido, lo cual genera un acercamiento a lo abstracto (pensamiento, opiniones, entre otras.) Estas representaciones han tenido diferentes explicaciones; una de ellas es la de Stuart Hall (1997), quien las define "como el uso de signos y símbolos cargados de significados que los individuos por medio de la apropiación y reorganización de elementos de cierto contexto cambian o dan nuevos significados a los objetos". Esto tiene que ver con el hecho de que las subculturas apropian objetos provenientes del mercado y los modifica e incorpora significados producidos por ellos (Hebdige, 1979). (Cita parafraseada).

Lo Importante de la posición de Hall (1997), es su planteamiento sobre cómo las representaciones se forman y transforman soportadas en un sistema que igualmente cambia; produciéndose así continuamente nuevos significados que exponen las conductas, identidades y las subjetividades del mismo sistema. Estos nuevos simbolismos son producidos a través de las prácticas significantes que se dan por un cambio en la sociedad y la relación con el sistema; puesto que la relación entre un significante y un significado es el resultado del sistema social y de convenciones específicas de cada sociedad y momentos históricos, pues el significado no está en el objeto o cosa, está en las personas que fijan los significados por medio de las prácticas que los originan a partir de la apropiación de elementos del sistema. Una prenda de vestir sola no significa nada, lo que 'dice' está en el sistema que la soporta; es por esto que los signos son arbitrarios pero sus significados los modifica el sistema, y este cambia constantemente dependiendo del contexto; o sea que las representaciones culturales son la imagen de algo y de alguien, expresado en las relaciones sociales, las cuales se basan en la acción comunicativa de los individuos al momento de relacionarse entre sí, y estas permiten la conformación de grupos a través de una suma 0 diferenciación de las identidades.

\section{RESULTADOS}

En la etapa de interpretación de los datos obtenidos se pudieron identificar las características particulares en cada uno de los grupos; además fue claro que la moda/vestido es una parte fundamental de la identidad de la subcultura, pero no imprescindible.

Así pues, en la subcultura punk existe un sentimiento de respeto hacia las demás personas, amor hacia los animales y la naturaleza, junto con una clara tendencia apolítica y antibélica. Algo importante es que la afiliación al grupo se da en los primeros años de la juventud, en los que se genera una afinidad o identificación con las letras de las canciones punk o con 
el estilo de un miembro de la subcultura.

Como se puede observar, es claro que existe un choque con algunos sectores de la sociedad, sobre todo en el ámbito laboral, donde los sujetos expresaron situaciones de rechazo o desaprobación. Ahora bien, hay casos de miembros de los grupos que usan prendas que se alejan del estilo típico punk; algunos son más extremistas en cuanto a los desgastes de las prendas, uso de elementos subversivos (que van en contra de lo establecido) o sacados de contexto, como los tornillos en los hombros de la chaqueta $u$ otros con ropa holgada, un poco más 'común', pero según ellos, esto también se debe a la necesidad de diferenciación que tienen, aun dentro del mismo grupo.

Respecto a la moda, la ven como parte de un sistema capitalista que alienta al consumo, en el que el diseñador no se preocupa por las implicaciones sociales y culturales de su práctica, y por estas razones están en contra de la misma. Por esta situación limitan el consumo de prendas de vestir al mínimo, pues crean su propio estilo a partir de ropa regalada o algunas prendas básicas sin marca, para posteriormente modificarla con pinturas, herrajes e intervenciones.

Este consumo mínimo se da aún con elementos de moda/ vestido que en algún momento fueron significativos dentro de la historia de la subcultura, como las botas 'Dr. Martens', que ahora son rechazadas por haber sido 'prostituidas' por el sistema de la moda, pues ya no tienen el significado obrero que tenían antes. Comprarlas ahora significaría una colaboración para construir otro imperio económico. En cuanto a los elementos de vestuario específicos y sus significados, puede decirse que la chaqueta es una de las piezas más importantes, pues contiene historias; entre más vieja, más valor tiene.

Con relación a los pantalones entubados, estos se usan para mostrar las botas "porque representan el trabajador, el obrero. El obrero siempre va a ir de botas adonde quiera que vaya." Otro elemento importante es el uso de cadenas y ganchos de nodriza; así pues, por una parte la cadena representa un símbolo de pertenencia obligada a la sociedad de consumo.

$Y$ en cuanto a los ganchos de ropa, funcionan como una forma de arreglarla de manera muy rápida, además de mostrar una imagen más ruda y funcional como una especie de atadura. Pero en realidad no parece existir un consenso o significación particular de este elemento.

Por otra parte, está la cresta o mohawk, que definen como una conmemoración a los indios que se levantaron contra la colonización inglesa; es una forma de defender y luchar por el territorio propio. Respecto a los colores, el rojo y el rojo negro hacen alusión al comunismo libertario; el verde hace alusión a la naturaleza y el blanco y negro a la igualdad, pues donde hay blancos, hay negros.

En resumen, se podría decir que aunque existen elementos de moda/vestido de la identidad particular de la subcultura Punk, en el fondo lo que se pretende es escapar de los estereotipos y una búsqueda de diferenciación.

\section{Elementos de identidad y moda/vestido en los metaleros}

En cuanto a la subcultura del Metal, se puede decir que hay una búsqueda por la libertad de expresión y autonomía de pensamiento, donde a veces hay rechazo a las manifestaciones religiosas, pues se consideran en contra de la naturaleza humana; además se declaran contra la sociedad de consumo y lo establecido; como dijo uno de ellos: ... "Que pereza seguir lo que siguen los demás para usted poder encajar y ser una buena persona; usted puede seguir siendo una buena persona sin tener que dejar sus cosas".

Así como sucede con los punkeros, los metaleros suelen unirse al grupo en la adolescencia, atraídos principalmente por la música, para luego adoptar el estilo característico de ellas. De igual manera, la asociación con la subcultura se da por la influencia o identificación con otros miembros de la misma.

Como ya se mencionó anteriormente, una vez existe el gusto por la música empieza a darse un cambio en la moda/vestido, el cual está caracterizado normalmente por jeans entubados, tenis Converse o deportivos de bota alta, camisetas con logos de bandas y a veces acompañados por chaleco de jean, chamarra de cuero y cinturón de balas.

Pero como pasa con los punkeros, este estilo puede tener algunas críticas y cierto rechazo por parte de la sociedad. Pero se hace visible que hay una mayor flexibilidad en el vestuario respecto a los punkeros frente a las diferentes situaciones sociales como el trabajo y eventos familiares como matrimonios, y que en algunos momentos se cede ante la presión social y terminan cambiando el estilo; así pues, el vestuario puede sufrir una separación de la identidad de estas personas, o sea, la identidad del metalero no depende todo el tiempo de la ropa. En cambio, en los espacios de ocio sí vuelven a mostrar esa parte de su identidad, de manera que en los momentos de descanso se expresan por medio de la moda/vestido.

Se hace visible que hay una mayor flexibilidad en el vestuario de los metaleros respecto a los punkeros, en diferentes situaciones sociales, como el trabajo y eventos familiares, y que en algunos momentos se cede ante la presión social y terminan cambiando el estilo. El vestuario puede dejar de ser parte de la 'identidad metalera' de estas personas, ya que como ellos dicen, "no hay necesidad de ser peludo para ser metalero". La identidad del metalero puede ser, en determinado momento, independiente de la ropa que usa.

Algo de resaltar es su observación con relación al hecho de que aunque cambien su vestuario de manera externa, siempre habrá algo que los conecte con el metal, ya sea una camisa interior o unos bóxeres negros, una manilla de cuero, un tatuaje. Así pues, el sentimiento de pertenencia a la subcultura puede ser íntimo, no existe necesidad de ser exteriorizado 
en todo momento, y siempre hay algún elemento de moda/ vestido que lo acompaña.

Respecto a la moda como sistema, no dieron ningún tipo de opinión en pro o en contra de ella, como sí lo hicieron los punkeros; pero es claro el importante papel que juega dentro del grupo, porque todas las historias siempre están de alguna manera relacionadas con el vestuario, ya sea cuando hablan de su trayectoria o de cómo asumen el Metal en su vida.

En cuanto al estilo específico del metalero, este consiste en camisetas, preferiblemente negras con logos de bandas o carátulas de discos para hacer visible lo que les gusta. Algo interesante es que algunos de ellos pintan sus propias camisetas, en una búsqueda de diferenciación, puesto que hay una necesidad de tener una identidad individual dentro del mismo grupo. Estas razones las convierten en un elemento simbólico, para depositar su identidad colectiva e individual.

En cuanto a los jeans, los usan negros o azules, entubados en su mayoría; también bermudas con estampado camuflado que implica una posición antiguerra. De hecho, algo muy importante en esta subcultura es la transposición de significados, pues toman elementos referentes a la violencia, como balas y camuflados, para hacer protesta contra ella, lo cual a veces es 'leído' de manera contraria por las personas que se encuentran por fuera del grupo.

En este tipo de prenda también pueden darse intervenciones como diferenciación; por ejemplo: El caso en el que un pantalón estaba recubierto de cinta industrial negra simulando cuero, expresa que, antes cuando se rompían los jeans en la entrepierna o cuando los tenis se despegaban, les ponían cinta para seguir utilizándolos. Cabe resaltar que para la intervención del pantalón se requirieron dos rollos de cinta, cada uno con un costo de USD 30; así pues, el precio se vuelve, de cierta manera, irrelevante frente al deseo de diferenciación.

Otro caso hace referencia a una foto de Metálica en la que uno de los integrantes aparece con los zapatos tenis con cinta enrollada. Siendo así, es notoria la influencia del vestuario de las bandas que escuchan, porque no solo se empiezan a vestir de manera similar a como lo hacen ellos, si no que a la hora de marcar una diferenciación dentro del grupo también los tiene como punto de referencia. Parte de su vestuario son las chamaras o chaquetas de cuero, camisas leñadoras, tenis Converse o de bota, alta similares a los que se usaban para jugar baloncesto en los años 80; los chalecos de jean, influenciados por el Trash Metal, que se usan decorados con parches alusivos a las bandas y taches de níquel, además del pelo largo, que es uno de los elementos más representativos, pero que no siempre está presente en el estilo de lo metaleros, porque no todos lo usan de esta manera; algunos eligen tener el pelo corto.

Otro elemento muy importante y notorio son las correas con balas, ya que figurativamente van en contra de la guerra, pero a su vez se vuelven un elemento paradójico con el pensamiento anticonsumo del metalero, pues estas pueden costar entre
USD 100 y 180 y suelen ser importadas de Estados Unidos. Esta contradicción también la evidencian cuando mencionan que lo que los diferencia con otros metaleros es el hecho de invertirle dinero y tiempo a su apariencia, ya sea reformando el vestido, usando ropa en buen estado todo el tiempo y pagando para arreglarla, y que esta se ajuste bien al cuerpo.

En resumen, el estilo del metalero está influenciado por la música; las bandas representan la referencia primaria para su moda/vestido, y aunque haya una similitud dentro de sus integrantes, de una manera u otra buscan la diferenciación, así sea prodigando significativas cantidades de dinero y tiempo.

\section{Elementos de identidad y moda/vestido en los hip hopers}

Finalmente, al hablar de la subcultura del hip hop se puede decir que en ella hay un fuerte sentimiento de lucha y responsabilidad social, que se vuelca en algunos casos en labores con las comunidades donde viven. Así como en las otras subculturas, los jóvenes empiezan a ser parte de ella en la época de adolescencia gracias al gusto por la música, el ritmo y su mensaje social que se mantiene a lo largo de los años.

Además remiten su gusto por esta música porque es fácil de hacer. Muchos de los integrantes de esta subcultura llevan más de 10 años en el hip hop ya sea como $\mathrm{MC}^{2}$, DJ33 , breakdancers $^{4}$ o graffitero ${ }^{5}$. Estos ven el hip hop como una forma de vida, no solo como un estilo de ropa o un gusto por la música, sino como una posición frente a la vida. Algo muy particular de los hip hopers es que hay un cambio significativo en la moda/vestido que usan para la vida laboral y la que manejan para los espacios de ocio: “El ropero no hace al rapero; uno hay veces se estrella porque uno lo encuentra a él así elegante, pero más allá de la pinta hay un MC tremendo con mucho talento". Aunque algunos manifiestan que se les han presentado situaciones de crítica y censura. Además se nota la adopción de la subcultura como una forma de rechazo a la cultura parental; para diferenciarse frente a una realidad impuesta. Según Hebdige (1979), es uno de los rasgos principales de los grupos subcuturales.

En cuanto al estilo, resalta el uso de ropa sobredimensionada y de tendencia deportiva, como camisetas de equipos de baloncesto, gorras y bandanas o pañoletas, tenis, y por otra parte se encuentran las botas de obrero que tienen relación con lo social. Todo esto siguiendo la tendencia del hip hop de

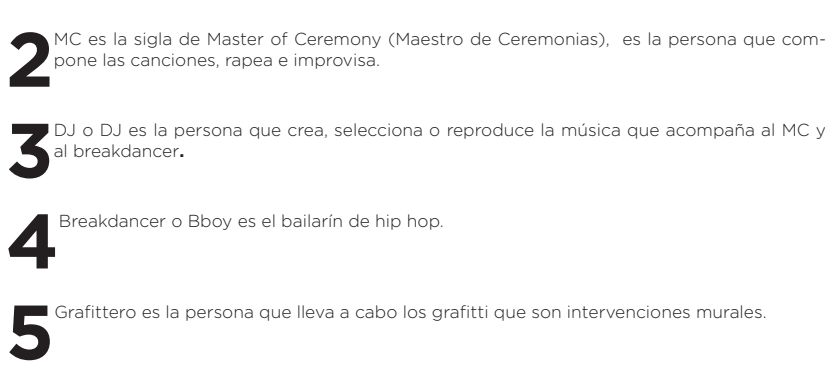


Estado Unidos con alusión a la comodidad, como un determinante en el uso de este tipo de ropa.

Algo clave que se repite en este grupo es la intervención del vestuario a falta de opciones accesibles en el mercado. No sucede como con los metaleros que era una cuestión de no encontrar lo que querían, si no por el costo. Hay también ropa no solo diseñada sino confeccionada por ellos mismos 0 sus familiares.

En resumen, los hip hopers tienen una fuerte conexión con lo social y la realidad de los lugares donde viven; es una subcultura/estilo de vida, donde la moda/vestido es importante pero no esencial, puesto que pueden prescindir de ella. También se observa que el vestuario no posee los mismos significados iníciales, con lo cual se intenta comunicar un todo, una forma de vida.

\section{RESULTADOS}

Al finalizar el proceso de investigación se puede ver, como dice Hebdige (1979), “una subcultura de este tipo se preocupa ante y sobre todo por el consumo. Opera exclusivamente en la esfera del ocio. (No me vestiría de punk para ir a trabajar, hay un momento y lugar para todo). Se comunica a través de productos, aunque los significados ligados a dichos productos se hallen deliberadamente distorsionados o abolidos".

Debido a que muchos de los accesorios del vestuario siguen teniendo significados similares a los que reposan en los libros de historia, como el uso de elementos militares en los metaleros para significar su posición contra la guerra y violencia, y los estampados en las camisetas de los punkeros como forma de protesta política, no tienen el mismo impacto en todos los contextos, puesto que el mensaje que quieren transmitir es incorrecto por su descontextualización dentro del sistema de representación de la ciudad.

Estos, más allá de comunicar, sirven como expresión visual y como una forma de comunicación, donde los integrantes de las diferentes subculturas pueden 'llamar' a sus semejantes; es decir, lo que expresan no es necesariamente transgresivo, pero sí una muestra de inconformidad. En conclusión, no son tan significativos como se pretende, porque la ciudad carece de un marco cultural igual al lugar donde fueron concebidos.

Entonces la correa de taches no está contra la guerra; es una señal de inconformidad y de querer salirse de la regularidad impuesta por la sociedad; por lo tanto, no hay un sentido verdadero ni una comunicación exitosa, pero es igualmente significativo para los individuos inmersos en ese sistema de representación. Esto lo confirma Hall (1997) cuando dice:

"Si la relación entre un significante y un significado es el resultado de un sistema de convenciones sociales específico de cada sociedad y de cada momento histórico -entonces todos los sentidos son producidos dentro de cada historia y cultura. No pueden estos sentidos estar finalmente fijados sino que siempre están sujetos a cambio, tanto de un contexto cultural a otro como de un período a otro. Por tanto no hay un 'sentido verdadero' que sea singular, incambiable y universal. "

Esta situación conlleva a una traslación de sentido en la que hay una constante producción de nuevos sentidos, de nuevas interpretaciones.

Así, el significado de origen social de la ropa sobredimensionada de un hip hoper no se entiende como un uso por necesidad; ni la utilización de cintas industriales en los pantalones de un metalero como una forma anticonsumo al no querer comprar unos nuevos, si no como una opción que se tomó de mostrar una idea o un estilo de vida.

Podemos concluir que es una muestra del contexto pereirano, ya que estos jóvenes crecieron en una sociedad relativamente homogénea, dentro de un espacio donde la diversidad de expresión era reducida y los obligó a unirse con otros para conformar un lugar donde pudiesen ser ellos mismos y lograr diferenciarse, más allá de estar afiliados a una ideología. Realmente se vuelve determinante el tipo de subcultura a la cual se pertenece a través de la música que más les agrada y los identifica de alguna manera, y es aquí donde los significados se desdibujan porque la afiliación a los grupos se da por un gusto musical más no por una ideología, de manera que lo que significa la ropa es algo secundario, pues esta se usa principalmente porque está relacionada con la música que escuchan.

¿Se podría decir que hay una falta de significado en los elementos de moda/vestido de las subculturas de Pereira?

No, pero el asunto es que se trata de una nueva construcción de significados, y de esta manera, los elementos de vestuario adquieren expresiones de afiliación a un grupo por inconformismo frente a la sociedad, o a una idea que se les ha vendido de cómo debe ser un pereirano, cómo debe actuar o vestirse. Seguramente por eso cuando se les explicaba la naturaleza de este proyecto de investigación no sentían agrado sino hasta que se les indicaba que su objetivo era mostrar otra cara de la ciudad; evidenciar las cosas diferentes que acontecían, y quiénes eran los otros que no solo existen sino que habitan la misma ciudad.

Por último, es importante evidenciar, que esta situación hace que la subcultura en Pereira esté unida en unos mismos espacios; compartan lugares de ocio, sean amigos, hagan conciertos de diferentes géneros en el mismo lugar y día, que se desdibujen las líneas de los grupos específicos y todos se conviertan en una sola subcultura. 


\section{REFERENCIAS BIBLIOGRÁFICAS}

Álvarez, G. (2007). Hip Hop: Más que calle. Buenos Aires: Era Naciente.

Aristizábal, C.A. (2008). Teoría y metodología de la investigación: Guía didáctica y módulo. Recuperado de http:// www.funlam.edu.co/administracion.modulo/NIVEL06/ TeoriaYMetodologiaDeLaInvestigacion.pdf

Arroyo, M. F. (2011). La moda en las tribus urbanas. Cacas: Universidad Católica Andrés Bello. Recuperado de http:// biblioteca2.ucab.edu.ve/anexos/biblioteca/marc/texto/ AAS2388.pdf

Ávila-Toscano, J., Gutiérrez, B. \& Pérez, J. (2011). Indicadores estructurales y conglomerados de actores en la red social de una subcultura urbana. Revista Colombiana de Psicología, 20 (2), 193-207. Recuperado de http:// www.revistas.unal.edu.co/index.php/psicologia/article/ view/17094

Brown, L. (2009). Hip to the game - dance world vs. music industry: The battle for Hip Hop's legacy. Movement Magazine, 18. Recuperado de http://www.movmnt.com / monsters -of-hip- hop-2_003332.html

Bucholtz, M. (2002). Youth and cultural practice. Annual Review of Anthropology 3,525-552. Recuperado de la base de datos de Jstore, http://www.jstor.org/stable/4132891

Buckingham, D. (2008). Introducing identity: Youth, identity and digital media. Series on Digital Media and Learning (pp. 1-24) Cambridge: The MIT Press.

Caffarelli, C. (2008). Tribus urbanas: Cazadores de identidad. Buenos Aires: Grupo Editorial Lumen.

Davis, F. (1992). Fashion, culture and identity. Chicago: The University of Chicago Press.

Dunn, S., McFayden, S. \& Feldman, S. (productores), Dunn, S. \& McFayden, S. (directores) (2005). Metal: A headbanger's journey .[Cintacinematográfica]. Canadá: SevillePictures\& Warner Home Video.

Feixa, C. (1996). De culturas juveniles al estilo. Nueva antropología, revista de ciencias sociales, 50, 71-89. Recuperado de la base de datos REDALYChttp://www.juridicas.unam. $\mathrm{mx} /$ publica/librev/rev/nuant/cont/50/cnt/cnt4.pdf

Garcés, A, Tamayo, P. \& Medina, J. D. (2007). Territorialidad e identidad Hip Hop raperos en Medellín. Revista Anagramas, Volumen 5 (10), pp. 125-138. Recuperado de http://cdigital.udem.edu.co/ARTICULO/ A082000102007151992/ARTICUL08.pdf

Gilroy, P. (1998). Los estudios culturales británicos y las trampas de la identidad. En J. Curran, J., Morley, D. \& V. (Ed.), Estudios culturales y comunicación: Análisis, Producción y Consumo Cultural de las Políticas de Identidad y el Posmodernismo. (63-83) . Barcelona: Paidós.

González, M. et al. (2012). Identities through fashion: A multidisciplinary approach. Londres: Berg Publishers.
Hall, S. \& Jefferson, T. (1976). Resistance through rituals: Youth subcultures in post-war-Britain. Birmingham: Routledge.

Hall, S. (1997). Representation: Cultural representations and signifying practices. Londres: SAGE Publications Ltd.

Hebdige, D. (1979). Subcultura: El significado de estilo. (C. Roche, Trad.). Barcelona: Paidós.

Hebdige, D. (1999). The function of subculture. En S. During (Ed.), The Cultural Studies Reader (pp. 441-450). New York: Routledge.

Kawamura, Y. (2011). Doing research in fashion and dress: An introduction to qualitative methods. Londres: Berg Publishers.

Kuper, H. (1973). Costume and identity. Comparative studies in society and history, 15 (3), 348-367. Cambridge: Cambridge UniversityPress.

Lipovetsky, G. (1996). El Imperio de lo efímero: La moda y su destino en las sociedades modernas (5a ed.). (F. Hernández y C. López, Trad). Barcelona: Anagrama.

Lurie, A. (1994). El Lenguaje de la Moda (2a ed.). (F. Inglés, Trad.). Barcelona: Ediciones Paidós Ibérica.

Machado, A. (1998). Representación, identidad, virtualidad. VI Congreso ALAIC: Asociación Latinoamericana de Investigadores de la Comunicación. Recuperado de www.eca. usp.br/associa/alaic/Congreso1999/.../Ada\%20Cristina. doc

Macías, H. (2008, 15 de diciembre). Representación social y cultural de las identidades, su estratificación y papel en el mercado laboral. [Web log post]. Recuperado de http:// conceptualdelacultura.blogspot.com/2008/12/representacin-cultural-y-social-de-las.html

McFayden, S. (productor), Dunn, S. \& McFayden, S. (directores) (2008). Global metal.[Cintacinematográfica].Canadá: Banger Productions.

Narumi, H. (2010). Street style and its meaning in postwar Japan. Fashion Theory, 14 (4), 415-438. Londres: BergPublishers.

Observatorio de Tendencias de la Cámara de Comercio de Bogotá (2009). Perfiles de consumidores. Recuperado de http://camara.ccb.org.co/documentos/4548_28_07_09_ perfiles_consumidores.pdf

\section{FORMA DE CITAR ESTE ARTÍCULO}

Uribe Restrepo, N. (2014). Identidad, estilo y subcultura en Pereira: la moda/vestido en el metal, punk y hip hop. Revista Arte y Diseño Facultad de Arquitectura, Arte y Diseño, Universidad Autónoma del Caribe, Barranquilla. ISSN 1692- 8555 Vol. 12 (N.1) PP. 14 - 20 